\title{
Germanica
}

\section{Vrais et faux malentendus. L'Homme difficile de Hugo von Hofmannsthal}

Echte und unechte Mißverständnisse - Der Schwierige von Hugo von

Hofmannsthal

\section{Geneviève Roussel}

\section{OpenEdition}

\section{Journals}

Édition électronique

URL : http://journals.openedition.org/germanica/1288

DOI : $10.4000 /$ germanica. 1288

ISSN : 2107-0784

\section{Éditeur}

Université de Lille

\section{Édition imprimée}

Date de publication : 30 juin 1998

Pagination : 19-31

ISBN : $9770984263203-22$

ISSN : 0984-2632

Référence électronique

Geneviève Roussel, «Vrais et faux malentendus. L'Homme difficile de Hugo von Hofmannsthal », Germanica [En ligne], 22 | 1998, mis en ligne le 31 janvier 2012, consulté le 06 octobre 2020. URL http://journals.openedition.org/germanica/1288; DOI : https://doi.org/10.4000/germanica.1288

Ce document a été généré automatiquement le 6 octobre 2020.

(c) Tous droits réservés 


\title{
Vrais et faux malentendus. L'Homme difficile de Hugo von Hofmannsthal
}

\author{
Echte und unechte Mißverständnisse - Der Schwierige von Hugo von \\ Hofmannsthal
}

Geneviève Roussel

1 Le début du vingtième siècle et surtout la première guerre mondiale ont, selon de nombreux écrivains européens, engendré des hommes nouveaux, différents, dont ils firent les personnages de leurs fictions romanesques ou théâtrales : soit égoïstes et violents, soit psychologiquement labiles, dépourvus de caractère, sans projet, dépossédés de toute certitude. Les écrivains d'Europe centrale tout particulièrement, de Kafka à Doderer, de Hasek à Kundera, proposent des personnages inadaptés au monde ou des «hommes sans qualités » (Mann ohne Eigenschaften), selon la formule de Musil. Hofmannsthal avait donné, d'abord, à sa comédie de l'époque de la guerre le titre révélateur d'Homme sans dessein (Mann ohne Absicht), mais il choisit finalement - en vertu de sa volonté de discrétion et d'ironie - le titre, prémonitoire, de L'Homme difficile. Karl Kraus, plus brutal, affirma que la grande guerre est apocalypse, que ni les humains, ni Dieu, ni l'histoire ne peuvent la maîtriser et que les hommes vivent donc Les Derniers jours de l'humanité (Die letzten Tage der Menschheit). Victimes d'un vide des valeurs (Wertvakuum que Broch analyse si bien à propos de Hofmannstahl) sans précédent, les hommes du vingtième siècle ne trouvent pas davantage refuge auprès de leurs semblables: la crise des relations entre les individus dévoile les béances qui les séparent; béances que colmatèrent ultérieurement, pour les plus faibles, de nouvelles doctrines grégaires, absolument néfastes; béances intégrées pour d'autres, voire magnifiées, mais synonymes d'isolement.

2 Dans l'œuvre de Hofmannsthal les textes de fiction ayant pour cadre l'époque contemporaine et Vienne sont très peu nombreux. Se privant de ses distanciations coutumières du temps, du lieu, du conte ou du mythe, il livra pourtant avec L'Homme difficile (1919) 1 une image de la Vienne, de l'Europe de son temps, et classa cette pièce dans la série des comédies (Lustspiele) - malgré son titre et l'amertume du bilan -, grâce au suprême écran de l'heureux dénouement molièresque qu'il lui imposa. 
Ma comédie est une comédie sociale, elle se passe aujourd'hui; dans le grand monde autrichien, grand monde transposé intellectuellement toutefois, tel que moi je le vois ${ }^{2}$.

3 Cette comédie sociale se passe donc vers la fin de la guerre dans l'aristocratie viennoise, flanquée de ses satellites habituels: domesticité, érudits, noblesse étrangère. Les rencontres s'y font, les intrigues s'y tissent selon les schémas traditionnels de la vie transposée au théâtre, dans le respect de codes fiables. Elles investissent les lieux clos protecteurs d'où l'on ne voit pas le reste du monde, qui n'a pas été convié et ne saurait intervenir que par effraction: le bureau très protégé du personnage principal dans le premier acte, la maison Altenwyl et ses salons pour la soirée mondaine du deuxième, son grandiose vestibule, lieu fatidique des chasses-croisés, pour le troisième. Cet univers est, en apparence, resté policé, la guerre pourtant, - sa discrète présence périphrastique -, modifie, détruit, réorganise parfois, selon des normes encore inconnues, les relations entre les individus, entre les individus et la société. Même s'ils pensent pour la plupart avoir retrouvé ou restauré le monde d'autrefois - la grande soirée en serait une des parfaites incarnations - les protagonistes sont porteurs, souvent à leur insu, de cicatrices, de dégoûts, de doutes, ou au contraire bardés, en guise d'idéologie, d'une toute fraîche suffisance qui devrait leur permettre de dominer ce monde épuisé.

Peut-être n'aurais-je jamais pu représenter avec autant d'amour la société que la pièce représente, l'aristocratie autrichienne, son charme et sa qualité, qu'en l'instant historique où elle, qui récemment encore était une donnée, une force, se dilue doucement, fantomatiquement, pour n'être plus rien, comme un dernier tout petit nuage de brume le matin ${ }^{3}$.

4 Étudiant lui-même a posteriori les lignes de force de ses œuvres, Hofmannsthal rapprocha, comme faisant partie d'une série, L'Aventurier, Le Chevalier à la rose et L'Homme difficile. Les critiques unanimes ont toujours qualifié de chefs-d'œuvre Le Chevalier à la rose et L'Homme difficile, ces deux comédies aux nombreux thèmes communs, entre gens bien élevés, ancrées en outre dans un même monde viennois. Certes le dix-huitième siècle du Chevalier à la rose a laissé son lumineux vernis dans l'art de la conversation du vingtième siècle, mais le langage constellé de termes français, qui était celui des Lumières et du bonheur, est toutefois devenu un langage hybride, par trop redevable à l'idiome de l'ennemi. Ce langage qui jadis débouchait sur l'action entraîne aujourd'hui des "méprises chroniques » («chronische Mißverständnisse ») que seul le silence peut éviter, que seuls de rarissimes dialogues authentiques peuvent constater et dépasser.

5 «La parole est à l'origine de toutes choses", affirme l'homme difficile. C'est essentiellement par la parole que s'expriment - nous sommes au théâtre - les relations entre les individus. Derrière le charme d'une langue virtuose du demi-mot se distinguent trois langages différenciés par leur nature plus que par leur niveau (il en va de même pour les silences !) : la conversation, acide, lénifiante, hyperbolique..., souvent chargée de montrer au spectateur comment progresse l'action, à l'insu des protagonistes eux-mêmes; les dialogues, peu nombreux, exceptionnellement longs, face à face où les individus se dévoilent lentement, comme à tâtons ; et surtout, pauvres tentatives pour éviter refus et méprises, preuves du solipsisme d'individus étrangers les uns aux autres comme à eux-mêmes, les messages transmis par intermédiaire. Paradoxalement chacun semble conscient qu'il va à l'échec s'il présente personnellement sa requête, quelle qu'elle soit, et charge donc un messager de la 
démarche. L'homme difficile en particulier, qui croit moins que jamais à la fiabilité des paroles, s'estime chargé, de par sa place dans la société, ou est chargé par autrui de plusieurs de ces missions, alors même qu'il est incapable de définir ses propres vœux. La pièce est une suite de ces intercessions, des quiproquos qu'elles entraînent, jusqu'à l'étonnante intervention de la jeune comtesse qui prend en mains son propre destin et fait fi du schéma convenu de la demande en mariage.

Le constat de Neuhoff, l'étranger de bonne naissance, qui, introduit dans l'univers viennois, le jauge froidement, semble confirmé par la pièce :

ici personne ne fait partie $\mathrm{du}$ monde réel, celui où se règlent les crises intellectuelles de notre siècle.

7 De fait les crises et leurs dénouements sont ici d'abord individuels.

8 Le comte Hans Karl Bühl, de retour du front, retrouve son monde policé d'avant la guerre, mais l'homme difficile qu'il était naguère pour son entourage ne se sent plus capable d'y reprendre sa place et fuit toute vie sociale pendant des semaines. En une journée cependant, celle de la pièce, il franchit le seuil d'une nouvelle vie, alors qu'il se dit définitivement convaincu de l'inadéquation du langage, voire même de son « indécence »; il met de l'ordre dans son univers quotidien (avec ses serviteurs, son secrétaire, sa famille), met un terme à sa liaison de pure galanterie avec la comtesse Hechingen, lui démontre qu'elle doit reconnaître l'amour de son mari, dont il est devenu l'ami pendant la guerre, et former dorénavant avec lui un vrai couple; persévère dans son refus de participer - par des discours - à la vie politique; plaide auprès de la comtesse Helene, fille de son ami Altenwyl, la demande en mariage de son neveu Stani. Mais dans son dialogue avec Helene il ne pourra s'empêcher de lui livrer sa plus profonde expérience pendant la guerre - la vision, lors d'un ensevelissement, de ce qu'aurait dû être sa vie : le bonheur dans l'union avec Helene -, confidence qui conduit celle-ci à l'amener de force à reconnaître leur amour réciproque et à conclure la journée par leurs fiançailles.

Dans L'Homme difficile l'aristocratie viennoise n'a pas encore rencontré l'Histoire.

La guerre n'a apparemment pas anéanti, pas même rendu ridicules salons et intrigues mondains; elle semble être une parenthèse, on ne la mentionne qu'allusivement (le terme lui-même n'apparaît que deux fois et seulement dans la bouche de la jeune génération ${ }^{3}$ ). Bien sûr ceux qui y participèrent furent tous courageux et dignes ! Elle les fit vivre une autre vie, partager le pain, vénérer les supérieurs et sourire aux visiteuses des hôpitaux. Mais personne n'estime transposable l'expérience de la fraternité du front : la guerre c'était « là-bas ", " au loin », " ailleurs », dit tout un chacun, reléguant dans cet ailleurs imprécis ( draußen») les champs de bataille hors du monde protégé des salons, de la ville, voire de l'Empire.

11 La guerre a pourtant contraint ceux qui y participèrent (presque tous les hommes de la pièce) à vivre hors des critères et références habituels. Dans la solitude et parfois la peur c'est d'abord à eux-mêmes qu'ils ont été confrontés : d'où crises et révélations. Lejeune neveu Stani se vantera seulement d'avoir découvert son inaltérable bonne santé. Hechingen, alors qu'il passait pour falot, fait preuve de courage et de sérénité devant la mort, et devient pour Hans Karl un indéfectible ami. Le secrétaire de Hans Karl, ayant perdu son meilleur ami à la guerre, juge de son devoir d'épouser la fiancée de cet ami. Hans Karl enfin a eu, grâce à la guerre et la mort aux aguets, révélation de "vérités enfouies ", la découverte de ce qu'est réellement un homme, et une « leçon de bonheur » à l'heure de la mort probable. 
12 Revenus du front, les hommes qui ont participé à la guerre voudraient reprendre leur vie d'avant la parenthèse, éventuellement préciser leur place sur l'échiquier de la vie sociale, sans pour autant, si l'on excepte les spectateurs que sont l'étranger et le nouveau serviteur, valet à la Brecht, renverser, ni même analyser cette société. Elle est pourtant, telle qu'elle est peinte par Hofmannsthal, pour le moins étrange, propice aux malentendus, et en voie de dissolution. La solitude est individuelle, mais également sociale et rend difficile toute relation qui dépasse le simple savoir-vivre, rend ambigus les rôles respectifs. Dans cette pièce qui débouche, comme tant d'autres textes de Hofmannsthal, sur le mariage comme condition du bonheur de l'individu et base de l'organisation sociale on rencontre deux couples seulement, antithétiques: le futur couple de Hans Karl et Helene et le couple désuni depuis longtemps de Hechingen et sa femme. Antoinette Hechingen a été la maîtresse de Hans Karl pendant la guerre et aimerait perpétuer, voire officialiser cette liaison. En attendant le retour de Hans Karl elle s'est jetée dans les bras du neveu Stani, qui ressemble tant à son oncle!

Les deux familles symétriquement croisées de Altenwyl et sa fille Helene et de Crescence et son fils Stani, extrêmement succinctes, font jouer à leurs membres des rôles multiples, contradictoires, tant à l'intérieur de la famille que dans les relations avec les autres. Altenwyl est davantage homme politique que père d'Helene, qui, elle, est à la fois jeune fille courtisée de tous et, malgré son jeune âge, maitresse de maison accomplie. Crescence au contraire, mère de Stani et sœur de Hans Karl, veut à tout prix marier à son goût son fils adulé, qui souvent lui tient lieu de partenaire. Crescence a auprès de son frère le rôle de mère - elle l'a sauvé, enfant, de la noyade, lui donne des leçons de bonne conduite, s'inquiète pour sa santé, ses fréquentations -, tout en reconnaissant en lui le chef de famille, qu'elle charge, par exemple, de faire la leçon à son fils Stani et de sonder la comtesse Helene que Stani voudrait épouser. Hans Karl à son tour est, pour son neveu, à la fois un confident et un père...

Ajoutons qu'Helene et Hans Karl sont de lointains cousins, que leurs amis sont communs, que leurs domestiques respectifs se connaissent et savent tout de leurs maîtres, qu'ils fréquentent les mêmes cercles et ont les mêmes divertissements : le vaudeville n'est pas loin, avec ses lettres d'amour égarées, le mari berné, l'étranger profiteur, le bas-bleu et l'homme célèbre faisant assaut de ridicule !

15 L'élégant paravent de conversations toujours courtoises dissimule mal un univers déjà disparate. Les personnages de l'ancien monde, presque anachroniques, mais si charmants, qui font perdurer leurs valeurs communes, sont confrontés aux personnages résolument modernes, ceux qui croient à la force. Les seconds, sûrs de parvenir à leurs fins - le pouvoir, dans tous les domaines - dans de brefs délais, jugent les premiers.

Toute civilisation a ses fleurons: dignité sans prétention, distinction tempérée

d'une grâce infinie, voilà le fleuron de cette vieille société.

16 Tel est l'avis faussement flatteur qu'exprime Neuhoff, l'Allemand aventurier, en présence de Hans Karl, dont l'antipathie se décèle à ses silences, ses gestes maniaques, ses réponses à double sens, son excessive courtoisie. Dès qu'il s'estime le plus fort, avec Helene par exemple, ou lorsqu'il devine qu'un invité partage son point de vue, a les mêmes ambitions que lui, le ton de Neuhoff change : la société viennoise, qu'il cherche à investir, en un typique réflexe de Haß-Liebe, est « un monde démodé » (altmodisch) « dénervé » (ohne Nerven), « sans force » (kraftlos), « indigne » (unwürdig), « vieux » (alt) tout simplement, ultime injure prometteuse. « Tous ces gens n'existent en réalité déjà 
plus. Ce ne sont plus que des ombres ». Quant à Hans Karl, le plus typique représentant de ce monde mort, et son concurrent auprès d'Helene, Neuhoff, après l'avoir accablé de compliments empoisonnés en lui rendant visite, dénonce hors de sa présence son autosatisfaction, son absence de volonté, de dignité, sa mollesse, son ambiguité, sa vanité, bref, "pour le caractériser en deux mots : un néant, absolu et prétentieux ». Lui, l'Allemand, est a contrario, comme il l'explique à Helene, la force, la virilité. Convaincu de son « droit du plus fort », il affirme que Helene l'épousera, obligatoirement fascinée par sa volonté.

Vous avez tout sacrifié à la beauté, même la force. Nous, dans notre Nord lointain, où les siècles nous ont oubliés, nous avons conservé la force.

Les relations entre Hans Karl et Neuhoff - double langage méprisant et silence contraint - sont au-delà déjà de toute crise. Intellectuellement chacun comprend parfaitement l'autre, mais ne peut, ne veut bouger d'un pas: deux conceptions du monde se font face, tôt ou tard la plus vivante l'emportera.

Font partie de l'ancien monde ceux, bien entendu, dont les noms parlent d'eux-mêmes : Altenwyl, qui met encore ses espoirs dans un système politique moribond, sa fille Helene, «le dernier et le plus beau fleuron » de ce monde dont elle accepte et incarne les conventions, mais jusqu'à l'instant seulement où son instinct de vie les balaie.

L'autre conception du monde, celle de la force, de la notoriété, du futur, a pour représentants peu nuancés des hommes "nouveaux », eux aussi caractérisés par leurs noms ou prénoms : Neuhoff, Neugebauer, le secrétaire de Hans Karl, l'homme célèbre, le Professeur Brücke, qui se voudrait reconnu par l'aristocratie, Vinzenz, le nouveau domestique. Ce dernier apparaît dès la scène d'introduction, où d'emblée s'opposent deux façons de servir : le domestique à l'ancienne, Lukas, énumère les manies de son maitre, affectueusement; le nouveau se jure de lui faire perdre dans les plus brefs délais ses fâcheuses habitudes et de le modeler à sa convenance. Hans Karl, trouvant le nouveau domestique «impossible», charge Lukas de le «jeter dehors». Les domestiques à l'ancienne, confidents, messagers, exécuteurs sont des doubles naïfs de leurs maîtres. Agathe, par exemple, la carriériste d'Antoinette Hechingen, est chargée de récupérer les lettres de la liaison avec Hans Karl : non seulement elle n'ignore rien de ce qui concerne les amours de sa maîtresse, mais elle y participe, elle use continuellement, lors de sa démarche, du « nous ", et fait référence au contenu, qui lui est familier et sentimentalement important, des lettres échangées. Ces domestiques remplissent avec fierté, dévotion presque, les missions qui leur sont confiées, implicites ou explicites, sans méfiance aucune. Leurs maitres au contraire, peu sûrs d'eux dans leurs rapports avec leurs semblables, croient organiser leurs démarches en tacticiens en chargeant un émissaire de leur monde de plaider leur cause : l'action et l'échec leur font peur. Crescence est ainsi l'avocat de son fils Stani, qui n'ose pas demander luimême Helene en mariage, auprès de Hans Karl, que Stani n'ose charger de sa demande ! Hans Karl sera donc l'avocat de Stani, selon le vœu de Crescence. Hechingen lui aussi choisit pour avocat auprès de sa femme Antoinette le même Hans Karl qui, après confirmation de leur rupture définitive, tente de lui démontrer que son devoir, et son bonheur, sont auprès de son mari. Ces curieuses relations triangulaires sont la preuve d'un extrême manque de confiance en soi dès qu'il s'agit de problèmes, de choix fondamentaux. Elles génèrent des dialogues aux circonvolutions complexes et aux sousentendus expressifs. Les hommes nouveaux n'ont au contraire aucune retenue dans la manifestation de leur volonté de puissance qui ne nécessite aucun intermédiaire : ce 
n'est pas d'amour que Neuhoff parle à Helene, il ne s'agit pas de convaincre, mais de l'emporter, tous les moyens étant bons, sans crainte du ridicule.

L'homme difficile, dans ses rapports avec lui-même et avec autrui, accumule, quant à lui, les atermoiements dûs à sa sensibilité. Les perplexités qu'engendre pour lui le langage, condition de l'action et du devenir, sont telles qu'il trouve refuge dans la solitude, signe d'élection. Les jeux de scène prévus par Hofmannsthal soulignent la tension sous l'extrême politesse. Une vingtaine de fois Hans Karl se montre «absent ", " hésitant ", «troublé ", " gêné ", " incertain »... " Nerveux », il « marche de long en large ", ouvre et claque les tiroirs de son bureau, fait de l'ordre dans la pièce, avant de "se reprendre "; une dizaine de fois Hofmannsthal note son silence, souvent orné de la fumée de sa cigarette; Hofmannsthal fait encore remarquer les nombreuses absences de réponse, et les multiples réponses que Hans Karl fait "à voix basse ", "à voix très basse ", ou comme " involontairement », et « sans regarder » son interlocuteur.

21 Hans Karl n'a pas changé pendant la guerre, il ne s'est pas endurci, constate sa sœur avec étonnement. Il n'a rien perdu de son charme, il est toujours « sans effort, un grand monsieur ", un «idéaliste", selon son neveu Stani, ses silences et hésitations contribuant pour une bonne part à ce charme. D'autres voient en lui un cynique, un séducteur à la Casanova, ou un hypocondriaque « puéril ».

Sa méfiance à l'égard du langage, indispensable dans les relations sociales, révèle l'état de crise dans lequel il vit depuis des semaines, sans voir, sans chercher d'issue. La vie sociale étant le lieu de "méprises chroniques » (« chronische Mißverständnisse ») qu'il ressent comme insupportables, il trouve à son malaise un dérivatif («Rekreation»), et une confirmation, dans le jeu exceptionnel d'un clown, mime muet dont Hans Karl vante l'absence de "dessein» ("Absicht»). À son malaise il oppose, expression déguisée et fictive de son désir, la vision qui, dans l'instant de son ensevelissement et de sa mort imminente, l'a envahi, réunissant une fois encore Eros et Thanatos. En trente secondes il a vu à quoi ressemble le bonheur : une vie où Helene était sa femme, " tout simplement, comme un fait accompli », en dehors de toute notion de temps. Mais le songe peut-il être une vie?

Deux femmes sont particulièrement sensibles à la crise de Hans Karl et l'interprètent comme passagère. Leur façon d'écouter, de prendre parti et d'intervenir se révèlent caractéristiques de la difficulté des relations entre hommes et femmes.

Après les reproches conventionnels de la femme séduite et abandonnée, Antoinette Hechingen, dans un long dialogue avec Hans Karl, refuse son projet de ressouder le couple Hechingen. Antoinette n'a rien de la grisette fin-de-siècle, rien non plus de la femme adultère traditionnelle des milieux mondains. Ce qu'elle revendique, non tant de Hans Karl que du monde entier, c'est le droit au plaisir, le droit au bonheur tels qu'elle les conçoit. La liaison avec Hans Karl lui a laissé un souvenir merveilleux, que personne ne pourra dégrader. Elle refuse de retrouver un mari dont elle ose dire qu'elle n'aime ni les mains, ni la façon d'être, un mariage dont la durée serait le seul ciment. Elle veut au contraire vivre dans l'instant, chaque fois que cet instant sera "beau». Hans Karl, affectant l'incompréhension, se retranche derrière ses convictions sur le rôle de la femme et propose à la "pauvre Antoinette » le schéma classique de l'amant devenant ami.

25 Helene Altenwyl, la plus jeune personne de la pièce avec ses vingt ans, sait opposer à Neuhoff une ironie polie; elle sait aussi orienter habilement le déroulement de la soirée, avec une parfaite maîtrise des usages et toute la subtilité de ses analyses 
psychologiques. Mais dès qu'elle devine la présence de Hans Karl, son trouble n'échappe à personne.

La première rencontre, très brève, de Helene et Hans Karl a lieu au début du deuxième acte: en quelques mots Hans Karl dit à Helene qu'il a quelque chose à lui dire, en ajoutant, alors que les invités se pressent autour des tables de jeu, cette remarque qui est un involontaire aveu: "Nous deux, nous ne jouons pas». Ce n'est que dans la dernière scène du deuxième acte que, selon le principe de retardement, Hans Karl et Helene peuvent reprendre leur conversation. Hans Karl répète : "J'ai à vous parler ». Dans un même souffle il formule ses réserves sur le langage, sur le poids souvent incommensurable des mots dans les relations entre les individus. Il reconnaît toutefois qu'il comprend parfaitement Helene, douée, dit-il, de " raison » ("Verstand »). À partir de là s'instaure un dialogue harmonieux, où Hans Karl montre involontairement - les mots sont donc bien révélateurs - combien il est séduit par Helene, par ses qualités profondes derrière sa beauté, pour conclure que celui qui vivrait à ses côtés connaîtrait le bonheur. Habile transition pour lui suggérer de ne pas épouser Neuhoff, mais plutôt Stani. Helene comprend que leur complicité va prendre fin et que cette scène est une scène d'adieu. Se dominant elle l'écoute raconter - il devient disert - ces quelques secondes où, comme certains mourants, il a eu la vision de sa vie: Helene y était sa femme et c'était le bonheur. Mais Hans Karl n'en tire pas la conclusion attendue et, sans avoir clairement présenté la demande en mariage de son neveu Stani, la quitte définitivement.

L'action n'a donc pas progressé. Stani et Hechingen sont toujours impatients de savoir si Hans Karl a réussi ses missions. Mais Hans Karl a quitté la soirée. Helene décide alors d'agir pour l'obliger à la choisir. Démarche insensée si l'on tient compte des relations entre hommes et femmes de son temps, de son milieu. Elle écrit une lettre d'adieu à son père et s'apprête à quitter la demeure pour chercher Hans Karl en ville, dans la nuit. À l'instant où, vêtue de son manteau de voyage, elle sort, elle rencontre Hans Karl revenu sur ses pas. Lui avouant alors qu'elle se préparait à le suivre, elle exige qu'il s'explique sur son retour... Elle emploie dorénavant des mots quasi indécents: «je sais », «je dois », «je veux». Elle passe au tutoiement et déclare sans détours: "je suis amoureuse de toi [...], je veux partager ta vie, ton âme, tout... »

C'est ainsi que se fiancent Hans Karl et Helene. En jeune femme moderne Helene a pris tous les risques. Mais Hans Karl, difficile dans ses relations avec ceux qui lui sont indifférents, ceux qui le gênent, ceux qu'il estime superficiels, n'a-t-il pas en réalité, conformément à la longue tradition théâtrale du quiproquo, parlé à Helene le langage des demi-aveux pour qu'elle les complète, le langage des faux malentendus pour qu'elle les dissipe?

29 Hofmannsthal pense, en 1918, que la tradition, l'ordre ancien du monde doivent se fondre peu à peu dans le présent, puis le futur, pour que soit préservée une certaine continuité. A la jeune génération de montrer qu'en conservant «certaines formes convenables et acceptées depuis des siècles ", même si le couple des fiancés est trop « bizarre » pour se tenir à ces formes, elle respecte une tradition à la valeur pratique et sociale : à la demande de Stani, les représentants des deux familles, le père d'Helene et la sœur de Hans Karl, s'embrassent, ce qui officialise les fiançailles. L'issue heureuse de la pièce, l'aisance parfaite de Stani pour s'adapter à des situations nouvelles et inattendues laissent deviner l'espoir auquel Hofmannsthal veut encore croire. 
La méfiance à l'égard du langage et de l'action, les méprises et malentendus qui jalonnent les vies, et partant la solitude des individus, sont encore dans L'Homme difficile tempérés par l'ironie de l'auteur, par le plaisir déjà nostalgique qu'il a à recréer littérairement la société qui l'entoure. Au fil du vingtième siècle la crise des relations prendra des formes de plus en plus variées, de plus en plus dures. Hofmannsthal dans sa dernière œuvre, inachevée, $L a$ Tour, mettra en scène le conflit père fils, éternel certes, mais ici porté à son paroxysme sous la forme d'un combat singulier entre un père et son fils que sépare une muraille réelle et symbolique à la fois.

31 Aujourd'hui Gregor von Rezzori, né en 1914, peut conclure ses Mémoires ${ }^{5}$ par un constat révélateur: les désordres du siècle ont fait la qualité propre de l'art de ce siècle :

Notre enfance s'est écoulée parmi des hommes socialement dérangés de leur position originelle, dans une époque historiquement dérangée, et elle a été remplie de désordres de toutes sortes; et le désordre conduit à la souffrance, et la souffrance à la plainte muette, là fleurit la poésie.

\section{NOTES}

1. Der Schwierige, Hofmannsthal écrivit sa pièce en 1917 et 1918 (derniers détails en 1919). La première impression date de 1920. La première représentation eut lieu le 8 novembre 1921 à Munich au Residenztheater (Hofmannsthal n'y assista pas).

Éditions accessibles: Der Schwierige, Lustspiel in drei Akten, in Lustspiele II, S. Fischer Verlag, 1965. Der Schwierige, in Dramen IV (Lustspiele), Fischer Taschenbücher.

En français : L'homme difficile, comédie en trois actes, deuxième édition révisée, Verdier, 1996 (première édition, 1992). Traduction et excellente postface de Jean-Yves Masson. La pièce a été jouée, dans la traduction révisée, à Paris du 21 mars au 27 avril 1997.

2. 19 septembre 1917, lettre à Rudolf Pannwitz.

3. 2 novembre 1919, lettre à Arthur Schnitzler.

4. Brücke : pont, intermédiaire...

5. Gregor von Rezzori, Neiges d'antan, Salvy éd., 1993, p. 263 (la graphie du terme dérangé est de l'auteur).

\section{RÉSUMÉS}

L'Homme difficile: comédie sociale et image littéraire de l'aristocratie viennoise à la veille de son effacement. La crise des relations entre individus et la crise des relations sociales est mise en évidence par le langage. Pour beaucoup le langage n'est plus sûr et ne débouche plus sur l'action : d'où les «malentendus chroniques » et le recours à l'intercession. Mais le monde de demain est déjà présent. Il prédit le retour des aventuriers, le pouvoir de la force, il suggère une nouvelle 
image de la femme... Le bonheur personnel et l'harmonie sociale peuvent-ils encore être trouvés, comme le suggère H.v.H., dans un vrai mariage?

Der Schwierige : soziales Lustspiel und literarisches Bild der österreichischen Aristokratie kurz vor ihrem Untergang. Die Krise der zwischenmenschlichen und gesellschaftlichen Beziehungen wird an der Sprache gemessen. Für viele ist sie nicht mehr glaubwürdig und führt nicht mehr zur Tat : daher die « chronischen Mißverständnisse » und die ständig verlangte Hilfe nach Fürsprache. Die Welt von morgen ist aber schon da: sie vertritt im voraus die Macht der Abenteurer und die Gewalt der Kraft; sie entwirft ein neues Bild der Frau. Sind individuelles Glück und soziale Harmonie noch in der höchsten Begegnung der Ehe zu finden, wie H.v.H. es hofft?

\section{AUTEUR}

\section{GENEVIÈVE ROUSSEL}

Université Charles-de-Gaulle - Lille 3 